\title{
BMJ Open Relationship between high-density lipoprotein cholesterol levels and endothelial function in women: a cross- sectional study
}

\author{
Yuji Takaeko, ${ }^{1}$ Shogo Matsui, ${ }^{1}$ Masato Kajikawa, ${ }^{2}$ Tatsuya Maruhashi, ${ }^{1}$ \\ Takayuki Yamaji, ${ }^{1}$ Takahiro Harada, ${ }^{1}$ Yiming Han, ${ }^{1}$ Haruki Hashimoto, ${ }^{1}$ \\ Yasuki Kihara, ${ }^{1}$ Eisuke Hida, ${ }^{3}$ Kazuaki Chayama, ${ }^{4}$ Chikara Goto, ${ }^{5}$ Yoshiki Aibara, ${ }^{6}$ \\ Farina Mohamad Yusoff, ${ }^{6}$ Shinji Kishimoto, ${ }^{6}$ Ayumu Nakashima, ${ }^{7}$ \\ Yukihito Higashi (1) 2,6
}

To cite: Takaeko Y, Matsui S, Kajikawa M, et al. Relationship between high-density lipoprotein cholesterol levels and endothelial function in women: a cross-sectional study. BMJ Open 2020;10:e038121. doi:10.1136/ bmjopen-2020-038121

- Prepublication history and additional material for this paper are available online. To view these files, please visit the journal online (http://dx.doi. org/10.1136/bmjopen-2020038121).

Received 02 March 2020 Revised 22 May 2020 Accepted 29 May 2020
Check for updates

(C) Author(s) (or their employer(s)) 2020. Re-use permitted under CC BY-NC. No commercial re-use. See rights and permissions. Published by BMJ.

For numbered affiliations see end of article.

Correspondence to Professor Yukihito Higashi; yhigashi@hiroshima-u.ac.jp

\section{ABSTRACT}

Objectives The purpose of this study was to evaluate the relationship between high-density lipoprotein cholesterol (HDL-C) levels and endothelial function in women.

Design Cross-sectional study.

Setting 22 university hospitals and affiliated clinics in Japan.

Participants 1719 Japanese women aged 17-90 years who were not receiving lipid-lowering therapy.

Measures We evaluated flow-mediated vasodilation (FMD) and serum levels of HDL-C. All participants were divided into four groups by HDL-C level: low HDL-C ( $<40 \mathrm{mg} / \mathrm{dL}$ ), moderate HDL-C (40-59 mg/dL), high HDL-C (60-79 md/dL) and extremely high HDL-C ( $\geq 80 \mathrm{mg} / \mathrm{dL})$.

Results Univariate regression analysis revealed a significant relationship between FMD and HDL-C $(r=0.12$, $\mathrm{p}<0.001)$. FMD values were significantly smaller in the low HDL-C group $(5.2 \% \pm 3.8 \%)$ and moderate HDL-C group $(5.2 \% \pm 3.8 \%)$ than in the extremely high HDL-C group $(6.7 \% \pm 3.4 \%)(p=0.024$ and $p=0.003$, respectively), while there was no significant difference in FMD between the high HDL-C group and the extremely high HDL-C group. Multiple logistic regression analysis did not show a significant association between HDL-C levels and FMD. Conclusions Endothelial function increased in relation to HDL-C levels. However, there was no association of HDL-C levels with endothelial function after adjustment of traditional cardiovascular risk factors in women. Trial registration number UMIN000012950; Results.

\section{INTRODUCTION}

Several observational studies have shown that high-density lipoprotein cholesterol (HDL-C) concentration is inversely associated with coronary heart disease. ${ }^{1-3}$ Serum HDL-C levels are also inversely associated with the risk of ischaemic stroke. ${ }^{45}$

It is well known that the atheroprotective effect of HDL is largely due to reverse cholesterol transport, which is transportation of excess cholesterol in the peripheral tissues to
Strengths and limitations of this study

- Our study included a large number of women who were not receiving lipid-lowering medicine and who underwent a flow-mediated vasodilation test.

- Our study shows the relationship between highdensity lipoprotein cholesterol levels and endothelial dysfunction in women.

- Owing to a lack of data, we did not perform detailed evaluation of menopausal status, including age at menopause and duration of menopause, and hormone therapy use in our study subjects.

- There may exist residual confounding for this crosssectional analysis.

the liver. Moreover, HDL induces endothelial nitric oxide synthase (eNOS) activation and stimulates nitric oxide $(\mathrm{NO})$ release in human endothelial cells. ${ }^{6}{ }^{7}$ In addition to reverse cholesterol transport and eNOS activation, HDL has been suggested to have the ability to inhibit low-density lipoprotein (LDL) oxidation, reduce inflammatory activation, attenuate endothelial cell apoptosis and stimulate endothelial repair processes. ${ }^{8-11}$

Recent studies have suggested that the atheroprotective features of HDL can be lost and become proatherogenic or dysfunctional in certain situations. ${ }^{12-14}$ Hirata et al ${ }^{15}$ reported that extremely high levels of HDL-C were significantly associated with increased risk of atherosclerotic cardiovascular disease mortality. Several studies have shown that a high HDL-C concentration is not always protective in postmenopausal or elderly woman. ${ }^{16-18}$ Large randomised clinical trials have shown that raising HDL-C levels using cholesteryl ester transfer protein (CETP) inhibitors did not prevent cardiovascular 
events. ${ }^{19-21}$ Some studies have shown that HDL function rather than HDL-C levels was associated with cardiovascular events. ${ }^{223}$ These studies have suggested that modulation of HDL-C levels alone is not a sufficient therapeutic target to prevent cardiovascular events.

Endothelial dysfunction and injury are established as a key early step of an atherosclerotic lesion and lead to cardiovascular disease progression. ${ }^{24}{ }^{25}$ Measurement of flow-mediated vasodilation (FMD) of the brachial artery has been used for assessment of endothelial function in clinical practice. FMD has been shown to be a significant predictor of cardiovascular events independent of cardiovascular risk factors. ${ }^{26} 27$

A few studies have demonstrated a protective effect of HDL-C on endothelial function. However, evidence regarding the relationship between HDL-C and endothelial function is very limited..$^{28}{ }^{29}$ We previously reported that an association of HDL-C levels with endothelial dysfunction was found in men in a community-based study. ${ }^{30}$ In men, high levels of HDL-C were independently associated with endothelial dysfunction. In the present study, we evaluated the relationship between HDL-C levels and endothelial function assessed by FMD in women not receiving lipid-lowering therapy.

\section{METHODS \\ Subjects}

A total of 10247 Japanese subjects were enrolled from the FMD-Japan registry $(n=7385)$ and from the Hiroshima University Vascular Function (HUVF) registry $(\mathrm{n}=2862)$. The study design of FMD-J is publicly available. ${ }^{31}$ In the HUVF registry, 2862 subjects who underwent a health check-up at Hiroshima University Hospital between August 2007 and August 2016 were enrolled. From these subjects, 2565 women were recruited (the remaining 7682 subjects were males). Subjects without information on HDL-C level $(n=171)$ and subjects with unclear images of the brachial artery interfaces $(n=1)$ were excluded. In order to eliminate the effect of treatment, subjects who were receiving lipid-lowering agents (eg, statins, proprotein convertase subtilisin/kexin type 9 inhibitors, ezetimibe, fibrates, bile acid sequestrants, eicosapentaenoic acid and niacin) ( $\mathrm{n}=674)$ were also excluded. Finally, 1719 subjects were enrolled in this study (online supplementary figure 1). The age range of the subjects was 17-90 years. Diabetes mellitus was defined according to the American Diabetes Association recommendations. ${ }^{32}$ Hypertension was defined as treatment with oral antihypertensive agents or systolic blood pressure of more than $140 \mathrm{~mm} \mathrm{Hg}$ and/or diastolic blood pressure of more than $90 \mathrm{~mm} \mathrm{Hg}$ without medication. Dyslipidaemia was defined according to the third report of the National Cholesterol Education Program. ${ }^{33}$ Smokers were defined as those who were current smokers. Coronary heart disease included angina pectoris, myocardial infarction and unstable angina. Cerebrovascular disease included haemorrhagic stroke, ischaemic stroke and transient ischaemic attack.
Cardiovascular disease was defined as coronary heart disease and cerebrovascular disease. Framingham risk score was calculated by points of risk factors: age, total cholesterol level, HDL-C level, systolic blood pressure, diabetes mellitus and smoking status. ${ }^{34}$

All of the participants were divided into four groups according to the definitions used in a previous study in Japan: low HDL-C $(<40 \mathrm{mg} / \mathrm{dL})$, moderate HDL-C (40-59 mg/dL), high HDL-C (60-79 $\mathrm{md} / \mathrm{dL})$ and extremely high HDL-C $(\geq 80 \mathrm{mg} / \mathrm{dL}) \cdot{ }^{35-37}$ Reduced HDL-C is defined as HDL-C $<40 \mathrm{mg} / \mathrm{dL}$ in WHO and Japanese criteria for metabolic syndrome. ${ }^{38}{ }^{39}$ The Framingham Study has shown that increased plasma HDL-C $(>60 \mathrm{mg} / \mathrm{dL})$ is a negative risk factor for cardiovascular disease. $^{40}$

Informed consent for participation in the study was obtained from all participants.

\section{Study protocol}

The subjects were instructed to fast overnight for at least 12 hours and avoid smoking, alcohol, caffeine and antioxidant vitamins on the day of the examination. ${ }^{30} 31$ Measurement of FMD was performed while each subject was in the supine position in a dark, quiet, air-conditioned room. Venous blood samples were obtained from the deep antecubital vein. FMD assessments were conducted in a blinded manner.

\section{Measurement of FMD}

The protocol for measurement of FMD has been previously described. ${ }^{30}{ }^{31}$ In the brachial artery, vascular response to reactive hyperaemia was assessed as an index of endothelial function. An occlusion cuff was wrapped around the forearm. We evaluated FMD using high-resolution ultrasonography and computer-assisted analysis software (UNEXEF18G, UNEX Co, Nagoya, Japan). This software is an automated edge detection system for measurement of brachial artery diameter. A longitudinal image of the brachial artery $(5-10 \mathrm{~cm}$ above the elbow) was scanned. The transducer was held at the point at which the clearest B-mode image of the anterior and posterior intimal interfaces can be obtained. Gain was set to obtain optimal images of the arterial lumen wall interface. When the tracking gate was placed on the brachial arterial lumen, the diameter was automatically tracked. After the baseline longitudinal image had been acquired, the occlusion cuff was inflated for $5 \mathrm{~min}$ ( $50 \mathrm{~mm} \mathrm{Hg}$ over systolic pressure). We recorded the longitudinal image of the artery until 3-5 min after cuff deflation. FMD was automatically calculated as the maximal percentage change in vessel diameter from the baseline value. The correlation coefficient between FMD analysed at the core laboratory and participant institutions was $0.84(\mathrm{p}<0.001)$. In our laboratory, the coefficient of variation for FMD was $10.1 \%$.

\section{Patient and public involvement}

Patients and the public were not involved in the design or planning of the study. 


\section{Statistical analysis}

All reported probability values were two sided. Continuous variables were summarised as mean $\pm \mathrm{SD}$ or medians (IQR). Comparison of variables among two or more groups by differences in the HDL-C levels was performed using the Wilcoxon test or Kruskal-Wallis test. Categorical values such as medications and medical histories were compared by means of the $\chi^{2}$ test. We preformed Tukey's post hoc test to compare the differences in FMD between groups. To identify independent variables associated with a lower quartile of FMD $(<3.8 \%)$, multiple logistic regression analysis was performed. In model 2, we adjusted for age, and in model 3, age, body mass index (BMI), hypertension, diabetes and current smoker were entered into the multiple logistic regression analysis. Receiver operating characteristic (ROC) analysis was performed to determine the cut-off value of HDL-C at which FMD would be lower than $3.8 \%$ (lower quartile). The subjects were divided into two groups according to the optimal cut-off value of HDL-C $(56 \mathrm{mg} / \mathrm{dL})$. We generated a set of matched cases (subjects with HDL-C $<56 \mathrm{mg} / \mathrm{dL}$ ) and controls (subjects with HDL-C $\geq 56 \mathrm{mg} / \mathrm{dL}$ ) using propensity score analysis. A logistic regression model was used to estimate the propensity of HDL-C $<56 \mathrm{mg} / \mathrm{dL}$ based on variables associated with HDL-C, including age, BMI, presence of hypertension and diabetes, triglycerides, lowdensity lipoprotein cholesterol, use of antihypertensive drugs (yes or no) and use of antihyperglycaemic drugs (yes or no). We created two well-matched groups based on clinical characteristics with these propensity scores using a calliper width of $0.2 \mathrm{SD}$ of the logit of the propensity score, and we compared the prevalences of endothelial dysfunction (defined as less than the lowest quartile of FMD in all participants). The data were processed using JMP V.13.0 software and Stata V.15. A probability value of $<0.05$ was considered statistically significant.

\section{RESULTS}

\section{Baseline characteristics}

The baseline characteristics of the 1719 subjects are shown in table 1 . Seven hundred and forty-one subjects (43.1\%) had hypertension, $629(36.6 \%)$ had dyslipidaemia, $145(8.4 \%)$ had diabetes mellitus, $59(3.4 \%)$ had previous cardiovascular disease and $133(7.8 \%)$ were current smokers.

\section{Relationship between HDL-C and endothelial function}

Univariate regression analysis revealed that HDL-C significantly correlated with FMD $(\mathrm{r}=0.12, \mathrm{p}<0.001)$ as shown in figure 1 . FMD values were $5.2 \% \pm 3.8 \%$ in the low HDL-C group, $5.9 \% \pm 3.7 \%$ in the moderate HDL-C group, $6.4 \% \pm 3.6 \%$ in the high HDL-C group and $6.7 \% \pm 3.4 \%$ in the extremely high HDL-C group. FMD values were significantly smaller in the low HDL-C group and moderate HDL-C group than in the extremely high HDL-C group $(p=0.024$ and $p=0.003$, respectively; figure 2$)$. When the extremely high HDL-C group was divided into an HDL-C of $80-99 \mathrm{mg} / \mathrm{dL}$ group and an HDL-C of $\geq 100 \mathrm{mg} / \mathrm{dL}$ group, FMD values were better in the HDL-C of $\geq 100 \mathrm{mg} /$ dL group than in the HDL-C of $80-99 \mathrm{mg} / \mathrm{dL}$ group (online supplementary figure 2 and online supplementary table 1). A lower quartile of FMD was less than $3.8 \%$ in this population. Multiple logistic regression analysis revealed that low HDL-C was not independently associated with a lower quartile of FMD after adjustment for age, BMI, presence of hypertension, diabetes and smokers (OR $1.13,95 \%$ CI 0.58 to 2.20 ; $\mathrm{p}=0.715$; table 2 ).

We next categorised subjects into four groups according to the quartiles of HDL-C. Baseline characteristics are summarised in online supplementary table 2 . FMD values were $5.5 \% \pm 3.7 \%$ in the low HDL-C group (quartile 1), $6.5 \% \pm 3.7 \%$ in the moderate HDL-C group (quartile 2), $6.4 \% \pm 3.6 \%$ in the high HDL-C group (quartile 3 ) and $6.7 \% \pm 3.4 \%$ in the extremely high HDL-C group (quartile 4). FMD values were significantly smaller in the low HDL-C group than in the moderate HDL-C group, high HDL-C group and extremely high HDL-C group $(\mathrm{p}<0.001$, $\mathrm{p}=0.002$ and $\mathrm{p}<0.001$, respectively; online supplementary figure 3). There were no significant differences in FMD between the moderate HDL-C group, high HDL-C group and extremely high HDL-C group. Multiple logistic regression analysis revealed that low HDL-C was not independently associated with a lower quartile of FMD (online supplementary table 3 ).

Results of ROC curve analysis to assess the sensitivity and specificity of HDL-C for predicting a lower quartile of FMD $(<3.8 \%)$ are shown in online supplementary figure 4 . Area under the curve value of the ROC curve was 0.58 , and the optimal cut-off value of HDL-C was $56 \mathrm{mg} / \mathrm{dL}$. We divided the subjects into two groups according to the optimal cut-off value of HDL-C $(56 \mathrm{mg} / \mathrm{dL})$. FMD was significantly smaller in the lower group than the other group $(\mathrm{p}<0.001)$. Baseline characteristics of subjects are summarised in (online supplementary table 4 ). There were significant differences between the two groups except for smokers and previous cardiovascular disease. We next evaluated the association between HDL-C and FMD in the propensity score-matched population. There were significant differences in total cholesterol, HDL-C and Framingham risk score. After matching for confounding factors, there were no significant differences in the values of FMD between the two groups ( $\mathrm{p}=0.581$; online supplementary table 5).

\section{DISCUSSION}

In the present study, there was a positive relationship between HDL-C levels and FMD in women who did not receive lipid-lowering therapy. The low HDL-C group $(<40 \mathrm{mg} / \mathrm{dL})$ showed the highest OR for a lower quartile of FMD $(<3.8 \%)$. Although the OR was highest in the low HDL-C group, statistical significance was diminished after adjustment for traditional cardiovascular risk factors. There was no significant difference in the values of FMD between the two groups divided by the optimal 
Table 1 Clinical characteristics of the subjects on the basis of HDL-C

\begin{tabular}{|c|c|c|c|c|c|c|}
\hline Variables & $\begin{array}{l}\text { Total } \\
(n=1719)\end{array}$ & $\begin{array}{l}\text { Low } \\
<40 \mathrm{mg} / \mathrm{dL} \\
(\mathrm{n}=53)\end{array}$ & $\begin{array}{l}\text { Moderate } \\
40-59 \mathrm{mg} / \mathrm{dL} \\
(\mathrm{n}=548)\end{array}$ & $\begin{array}{l}\text { High } \\
60-79 \mathrm{mg} / \mathrm{dL} \\
(\mathrm{n}=771)\end{array}$ & $\begin{array}{l}\text { Extremely high } \\
\geq 80 \mathrm{mg} / \mathrm{dL} \\
(\mathrm{n}=347)\end{array}$ & $\begin{array}{l}P \text { value } \\
\text { for rend }\end{array}$ \\
\hline Age, year & $52.6 \pm 14$ & $56.5 \pm 16$ & $54.2 \pm 15$ & $51.8 \pm 14$ & $51.3 \pm 13$ & $<0.001$ \\
\hline Body mass index, $\mathrm{kg} / \mathrm{m}^{2}$ & $22.3 \pm 3.7$ & $24.3 \pm 4.7$ & $23.7 \pm 4.2$ & $21.9 \pm 3.3$ & $20.4 \pm 2.5$ & $<0.001$ \\
\hline $\begin{array}{l}\text { Systolic blood pressure, } \\
\mathrm{mm} \mathrm{Hg}\end{array}$ & $123.2 \pm 19$ & $127.6 \pm 21$ & $127.6 \pm 20$ & $121.6 \pm 18$ & $119.2 \pm 19$ & $<0.001$ \\
\hline $\begin{array}{l}\text { Diastolic blood pressure, } \\
\mathrm{mm} \mathrm{Hg}\end{array}$ & $76 \pm 12$ & $77 \pm 12$ & $77 \pm 12$ & $75 \pm 12$ & $74 \pm 11$ & $<0.001$ \\
\hline Heart rate, bpm & $67 \pm 11$ & $72 \pm 13$ & $68 \pm 10$ & $67 \pm 12$ & $65 \pm 11$ & $<0.001$ \\
\hline Total cholesterol, mg/dL & $206 \pm 36$ & $184 \pm 41$ & $198 \pm 36$ & $206 \pm 35$ & $220 \pm 32$ & $<0.001$ \\
\hline Triglycerides, mg/dL & 99 (60 to 121$)$ & 164 (113 to 196$)$ & 124 (76 to 155$)$ & 89 (57 to 109$)$ & 71 (48 to 85 ) & $<0.001$ \\
\hline $\mathrm{HDL}-\mathrm{C}, \mathrm{mg} / \mathrm{dL}$ & $67 \pm 16$ & $35 \pm 3$ & $52 \pm 5$ & $69 \pm 6$ & $91 \pm 10$ & $<0.001$ \\
\hline LDL-C, mg/dL & $120 \pm 32$ & $116 \pm 40$ & $123 \pm 32$ & $120 \pm 32$ & $116 \pm 30$ & 0.018 \\
\hline Glucose, mg/dL & $98 \pm 22$ & $113 \pm 36$ & $102 \pm 23$ & $96 \pm 19$ & $94 \pm 18$ & $<0.001$ \\
\hline \multicolumn{7}{|l|}{ Medications, n (\%) } \\
\hline $\begin{array}{l}\text { Antihypertensive } \\
\text { therapy }\end{array}$ & $535(31.1)$ & $21(39.6)$ & $233(42.5)$ & $209(27.1)$ & $72(20.8)$ & $<0.001$ \\
\hline $\begin{array}{l}\text { Antihyperglycaemic } \\
\text { therapy }\end{array}$ & $82(4.8)$ & $9(17.0)$ & $42(7.7)$ & $20(2.6)$ & $11(3.2)$ & $<0.001$ \\
\hline $\begin{array}{l}\text { Framingham risk score, } \\
\%\end{array}$ & $5.4 \pm 5.3$ & $10.6 \pm 7.9$ & $8.1 \pm 6.5$ & $3.9 \pm 3.7$ & $3.7 \pm 3.6$ & $<0.001$ \\
\hline \multicolumn{7}{|l|}{ Medical history, $\mathrm{n}(\%)$} \\
\hline Hypertension & $741(43.1)$ & $34(64.2)$ & $314(57.3)$ & $290(37.6)$ & $103(29.7)$ & $<0.001$ \\
\hline Dyslipidaemia & $629(36.6)$ & $53(100.0)$ & $242(44.2)$ & $251(32.6)$ & 83 (23.9) & $<0.001$ \\
\hline Diabetes mellitus & $145(8.4)$ & $14(26.4)$ & $69(12.6)$ & $40(5.2)$ & $22(6.3)$ & $<0.001$ \\
\hline Smokers & $133(7.8)$ & $4(7.6)$ & $49(9.0)$ & $56(7.3)$ & $24(6.9)$ & 0.630 \\
\hline $\begin{array}{l}\text { Previous } \\
\text { cardiovascular disease }\end{array}$ & $59(3.4)$ & $4(7.6)$ & $20(3.7)$ & $27(3.5)$ & $8(2.3)$ & 0.248 \\
\hline FMD, \% & $6.3 \pm 3.6$ & $5.2 \pm 3.8$ & $5.9 \pm 3.7$ & $6.4 \pm 3.6$ & $6.7 \pm 3.4$ & $<0.001$ \\
\hline
\end{tabular}

Data are presented as mean \pm SD or median (IQR).

FMD, flow-mediated vasodilation; HDL-C, high-density lipoprotein cholesterol; LDL-C, low-density lipoprotein cholesterol.

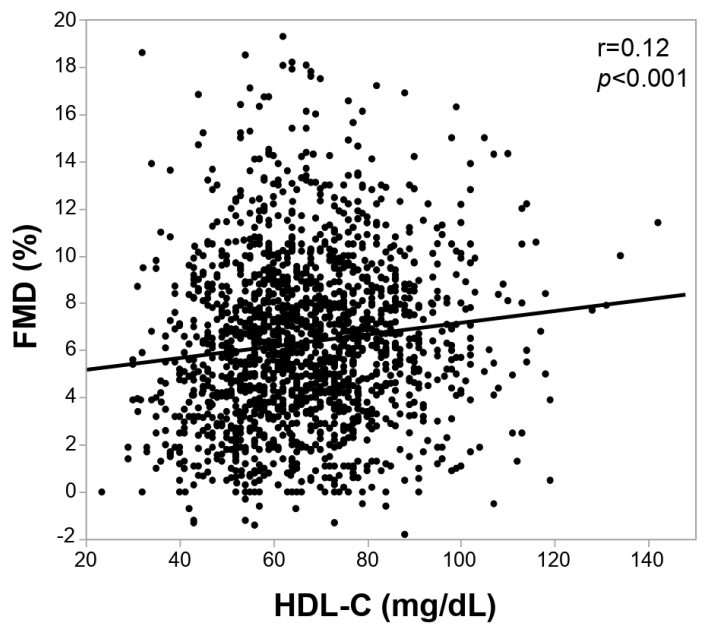

Figure 1 Scatter plots show the relationship between flowmediated vasodilation (FMD) and high-density lipoprotein cholesterol (HDL-C). cut-off value of HDL-C $(56 \mathrm{mg} / \mathrm{dL})$ after matching for confounding factors. These findings suggest that there is no association of HDL-C levels with endothelial function in women after adjustment of traditional cardiovascular risk factors.

Previous studies have shown that HDL-C concentration was inversely associated with coronary heart disease and ischaemic stroke. ${ }^{1-5}$ However, the precise mechanism by which low HDL-C causes cardiovascular complications remains unclear. Low HDL-C levels are often seen in patients with hypertension, hypertriglyceridaemia, insulin resistance and obesity. Indeed, in the present study, the values of triglycerides and glucose, age, BMI, prevalence of hypertension and prevalence of diabetes mellitus were higher in the low HDL-C group than in the other groups. It is possible that these confounding factors reflect endothelial function in subjects with low HDL-C. Low HDL-C and metabolic disorders coexist and may influence each other, leading to impairment of endothelial function. 


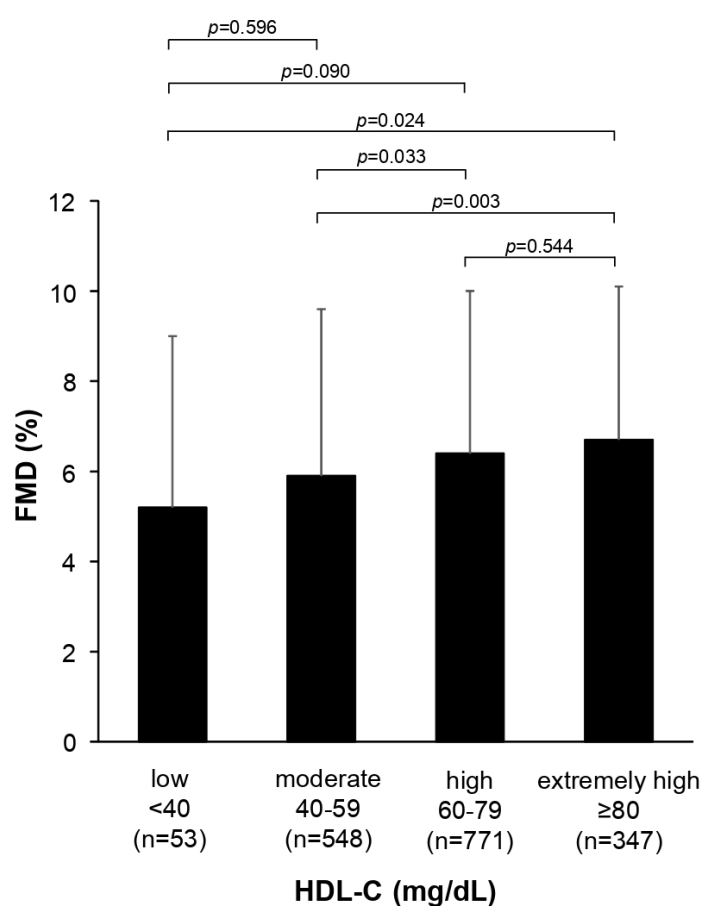

Figure 2 Bar graphs show values of flow-mediated vasodilation (FMD) in the groups of low levels of high-density lipoprotein cholesterol (HDL-C), moderate levels of HDL-C, high levels of HDL-C and extremely high levels of HDL-C. The error bars indicate SD.

In the WHO criteria for metabolic syndrome, reduced HDL-C is defined as HDL-C $<40 \mathrm{mg} / \mathrm{dL} .{ }^{38}$ It was shown in the Framingham Study that the increased plasma HDL-C $(>60 \mathrm{mg} / \mathrm{dL})$ is a negative risk factor for cardiovascular disease. ${ }^{40}$ In the present study, ROC analysis was performed to determine the cut-off value of HDL-C at which FMD would be lower than $3.8 \%$ (a lower quartile of FMD). The cut-off value derived from the results of ROC analysis was HDL-C $<56 \mathrm{mg} / \mathrm{dL}$. However, the area under the curve value was very small $(0.58)$, indicating poor discriminatory power, and the HDL-C cut-off value of $<56 \mathrm{mg} / \mathrm{dL}$ therefore has weak support.

The present study showed that FMD was significantly greater in subjects with extremely high HDL-C than in subjects with low HDL-C. However, extremely high HDL-C was not significantly associated with better endothelial function after adjustment for cardiovascular risk factors. Recent pharmacological studies have suggested that modulation of HDL-C levels alone is not a sufficient therapeutic target to prevent cardiovascular events. In large clinical trials, efforts to raise HDL-C levels by inhibition of CETP did not decrease cardiovascular outcomes despite increases in HDL-C. ${ }^{19-21}$ In our study, although the extremely high HDL-C group had a lower Framingham risk score than the scores in the other groups, extremely high HDL-C was not significantly associated with better endothelial function after adjustment for cardiovascular risk factors. These findings suggest that high HDL-C does not necessarily lead to good endothelial function even in individuals with low cardiovascular risk.

We previously reported that extremely high levels of HDL-C were independently associated with endothelial dysfunction in men who were not receiving lipid-lowering therapy. ${ }^{30}$ Interestingly, it is likely that there is a significant difference in the relationships between extremely high levels of HDL-C and endothelial function in men and women. In the present study, we divided the extremely high HDL-C group into an HDL-C of 80-99 mg/dL group and an HDL-C of $\geq 100 \mathrm{mg} / \mathrm{dL}$ group. Endothelial function was greater in the HDL-C of $\geq 100 \mathrm{mg} / \mathrm{dL}$ group than in the HDL-C of $80-99 \mathrm{mg} / \mathrm{dL}$ group in women. Possible reasons for the opposite results concerning the relationship between HDL-C levels and endothelial function in men and women may be differences in CETP activity and levels of oestradiol. It has been reported that CETP deficiency is one of the major causes of increased HDL-C levels in Japan. ${ }^{41}{ }^{42}$ Some single nucleotide polymorphisms (SNPs) in CETP had a differential effect on HDL levels and cardiovascular disease in men and women. ${ }^{4344} \mathrm{~A}$ haplotype consisting of the rs5883T and rs9930761CSNPs in CETP was shown to be associated with increased risk of progression to cardiovascular disease in men but not in women. ${ }^{43}$ Although gender differences in HDL function have not been elucidated, endogenous oestrogen presumably protects against atherosclerosis. It has been

Table 2 Multivariate analysis of the relationship between FMD and HDL-C

\begin{tabular}{|c|c|c|c|c|c|c|}
\hline \multirow[b]{2}{*}{ Variables } & \multicolumn{2}{|l|}{ Model 1} & \multicolumn{2}{|l|}{ Model 2} & \multicolumn{2}{|l|}{ Model 3} \\
\hline & OR $(95 \% \mathrm{CI})$ & $P$ value & OR $(95 \% \mathrm{CI})$ & $P$ value & OR (95\% Cl) & $P$ value \\
\hline \multicolumn{7}{|c|}{ HDL-C, mg/dL } \\
\hline $40-59$ & 1 (reference) & & 1 (reference) & & 1 (reference) & \\
\hline $60-79$ & $0.62(0.48$ to 0.80$)$ & $<0.001$ & 0.70 (0.53 to 0.92$)$ & 0.011 & 0.84 (0.63 to 1.12$)$ & 0.228 \\
\hline
\end{tabular}

Lower quartile of FMD indicate less than $3.8 \%$.

Model 1: unadjusted model.

Model 2: adjusted for age.

Model 3: adjusted for age, BMI, the presence of hypertension, diabetes and current smoking.

FMD, flow-mediated vasodilation; HDL-C, high-density lipoprotein cholesterol. 
suggested that HDL and oestradiol interact to stimulate eNOS activity and production of NO. HDL isolated from women stimulated eNOS activity, whereas HDL isolated from men had minimal activity for stimulation of eNOS activity. ${ }^{45}$ Oestradiol may have an impact on the effects of HDL on endothelial function. In addition to genetic variants and levels of oestradiol, environmental factors such as smoking, alcohol consumption, coffee intake and exercise contribute to the difference in the role of HDL-C in endothelial function in men and women.

Our results suggest that the circulating level of HDL-C lacks usefulness as an independent marker of endothelial function in women. It is well known that HDL inhibits atherogenesis through cholesterol efflux from peripheral tissues, activation of eNOS, inhibition of LDL oxidation, prevention of endothelial cell apoptosis and stimulation of endothelial repair processes. ${ }^{6-11}$ Several studies have provided evidence that the effects of HDL on endothelial function are highly heterogeneous. ${ }^{46}$ Antiatherogenic effects of HDL are impaired in patients with diabetes mellitus, coronary artery disease and chronic kidney disease. ${ }^{47}$ Although the precise mechanism by which endothelial effects of HDL are altered in patients with increased cardiovascular risk remains to be elucidated, it has been shown that an inflammatory response can cause alterations of HDL-C protein and lipid cargo. ${ }^{46}{ }^{48}$ It has been reported that isolated HDL-C from type 2 diabetes patients had reduced ability to stimulate eNOS activity $(-40 \%, \mathrm{p}<0.001)$ and to prevent nuclear factor kappa B (NF-KB) activation stimulated by tumour necrosis factor alpha in human microvascular endothelial cells $(-20 \%$, $\mathrm{p}<0.001) .{ }^{49}$ Recently, HDL function, especially cholesterol efflux capacity, has been used as an indicator of the relationship between HDL-C and cardiovascular disease. ${ }^{22} 23$ We should pay attention to HDL function rather than circulating levels of HDL-C.

There are some limitations in this study. First, we did not perform detailed evaluation of menopausal status, including age at menopause and duration of menopause, and hormone therapy use in our study subjects. Several studies have shown that a high level of HDL-C is not always cardioprotective in postmenopausal women. ${ }^{16}{ }^{17}$ It has been proposed that a decrease in oestrogen levels during the menopausal transition may lead to chronic inflammation. ${ }^{50}$ This systematic inflammation could potentially convert HDL-C to a dysfunctional form. In the present study, there was limited information on the precise menopausal status and hormone therapy use. To evaluate the association between endothelial function and HDL-C. levels over the menopausal transition, it is necessary to consider differences in HDL-C and FMD before and after menopause in the same subjects. Second, we were not able to evaluate lifestyles including alcohol drinking, detailed smoking status, diet and regular exercise, since there was limited information on lifestyles in our database. It has been shown that there is a positive gradient of HDL-C levels with alcohol consumption. ${ }^{51}$ Although the relationship between endothelial function and alcohol intake in women remains unclear, the amount of alcohol drinking might have an impact on the relationship between HDL-C and endothelial function. In addition, in the statistical analysis, consideration was only given to whether the subject was currently a smoker. Whether the subject is a current smoker as well as the frequency and number of cigarettes, such as pack years, are important when considering their effects on endothelial function. Finally, unfortunately, HDL-C functions were not evaluated. Further studies are needed to confirm the effects of HDL functions on endothelial function in a large clinical trial.

In conclusion, endothelial function increased in relation to HDL-C levels in women. However, there was no association of HDL-C levels with endothelial function in women after adjustment of traditional cardiovascular risk factors.

\section{Author affiliations}

${ }^{1}$ Department of Cardiovascular Medicine, Hiroshima University Faculty of Medicine Graduate School of Biomedical and Health Sciences, Hiroshima, Japan

${ }^{2}$ Division of Regeneration and Medicine, Medical Center for Translational and Clinical Research, Hiroshima University Hospital, Hiroshima, Japan

${ }^{3}$ Department of Biostatistics and Data Science, Osaka University Graduate School of Medicine, Osaka, Japan

${ }^{4}$ Department of Gastroenterology and Metabolism, Hiroshima University Faculty of Medicine Graduate School of Biomedical and Health Sciences, Hiroshima, Japan ${ }^{5}$ Department of Physical Therapy, Hiroshima International University,

HigashiHiroshima, Hiroshima, Japan

${ }^{6}$ Department of Cardiovascular Regeneration and Medicine, Research Institute for Radiation Biology and Medicine, Hiroshima, Japan

${ }^{7}$ Department of Stem Cell Biology and Medicine, Hiroshima University Faculty of Medicine Graduate School of Biomedical and Health Sciences, Hiroshima, Japan

Acknowledgements The authors would like to thank all patients who participated in this study. In addition, we would like to thank Miki Kumiji, Megumi Wakisaka, Ki-ichiro Kawano and Satoko Michiyama for their excellent secretarial assistance; FMD-J investigators of Takayuki Hidaka, MD, PhD; Shuji Nakamura, MD, PhD; Junko Soga, MD, PhD; Yuichi Fujii, MD, PhD; Naomi Idei, MD; Noritaka Fujimura, MD, PhD; Shinsuke Mikami, MD, PhD; Yumiko Iwamoto, MD; Akimichi Iwamoto, MD, PhD; Takeshi Matsumoto, MD, PhD; Nozomu Oda, MD, PhD (Department of Cardiovascular Medicine, Hiroshima University Graduate School of Biomedical Sciences, Hiroshima, Japan); Kana Kanai, PhD; Hraruka Morimoto, PhD (Department of Cardiovascular Regeneration and Medicine, Research Institute for Radiation Biology and Medicine, Hiroshima University, Hiroshima, Japan); Tomohisa Sakashita, MD, PhD; Yoshiki Kudo, MD, PhD (Department of Obstetrics and Gynecology, Hiroshima University Graduate School of Biomedical Sciences, Hiroshima, Japan); Taijiro Sueda, MD, PhD (Department of Surgery, Hiroshima University Graduate School of Biomedical Sciences, Hiroshima, Japan); Hirofumi Tomiyama, MD, PhD, FAHA; Akira Yamashina, MD, PhD (Department of Cardiology, Tokyo Medical University, Tokyo, Japan); Bonpei Takase, MD, PhD, FAHA (Division of Biomedical Engineering, National Defense Medical College Research Institute, Tokorozawa, Japan); Takahide Kohro, MD, PhD (Department of Cardiology, Tokyo Medical University, Tokyo, Japan); Toru Suzuki, MD, PhD (Cardiovascular Medicine, University of Leicester, Leicester, UK); Tomoko Ishizu, MD, PhD (Cardiovascular Division, Institute of Clinical Medicine, University of Tsukuba, Ibaraki, Japan); Shinichiro Ueda, MD, PhD (Department of Clinical Pharmacology and Therapeutics, University of the Ryukyu School of Medicine, Okinawa, Japan); Tsutomu Yamazaki, MD, PhD (Clinical Research Support Center, Faculty of Medicine, The University of Tokyo, Tokyo, Japan); Tomoo Furumoto, MD, PhD (Department of Cardiovascular Medicine, Hokkaido University Graduate School of Medicine, Hokkaido, Japan); Kazuomi Kario, MD, PhD (Division of Cardiovascular Medicine, Jichi Medical University School of Medicine, Tochigi, Japan); Teruo Inoue, MD, PhD (Department of Cardiovascular Medicine, Dokkyo Medical University, Mibu, Tochigi, Japan); Shinji Koba, MD, PhD (Department of Medicine, Division of Cardiology, Showa University School of Medicine, Tokyo, Japan); Kentaro Watanabe, MD, PhD (Department of Neurology, Hematology, Metaboism, Endocrinology and Diabetology, Yamagata 
University School of Medicine, Yamagata, Japan); Yasuhiko Takemoto, MD, PhD (Department of Internal Medicine and Cardiology, Osaka City University Graduate School of Medicine, Osaka, Japan); Takuzo Hano, MD, PhD (Department of Medical Education and Population-based Medicine, Postgraduate School of Medicine, Wakayama Medical University, Wakayama, Japan); Masataka Sata, MD, PhD (Department of Cardiovascular Medicine, Institute of Health Biosciences, The University of Tokushima Graduate School, Tokushima, Japan); Yutaka Ishibashi, MD, $\mathrm{PhD}$ (Department of General Medicine, Shimane University Faculty of Medicine, Izumo, Japan); Koichi Node, MD, PhD (Department of Cardiovascular and Renal Medicine, Saga University, Saga, Japan); Koji Maemura, MD, PhD (Department of Cardiovascular Medicine, Nagasaki University Graduate School of Biomedical Sciences, Nagasaki, Japan); Yusuke Ohya, MD, PhD (The Third Department of Internal Medicine, University of the Ryukyus, Okinawa, Japan); Taiji Furukawa, MD, $\mathrm{PhD}$ (Department of Internal Medicine, Teikyo University School of Medicine, Tokyo, Japan); Hiroshi Ito, MD, PhD (Department of Cardiovascular Medicine, Okayama University Graduate School of Medicine, Dentistry and Pharmaceutical Sciences, Japan); and Hisao Ikeda, MD, PhD (Faculty of Fukuoka Medical Technology, Teikyo University, Omuta, Japan)

Contributors YT and YuH: drafting the article and conception of this study; MK, TM, HH, SK, SM, TH, YiH, CG, YA, AN, FMY and TY: acquiring subjects and/or data; $\mathrm{EH}, \mathrm{KC}$ and $\mathrm{YK}$ : revising the article critically for important intellectual content. YuH is the guarantor of this work and, as such, had full access to all the data in the study and takes responsibility for the integrity of the data and the accuracy of the data analysis.

Funding Grant-in-Aid for Scientific Research from the Ministry of Education, Science and Culture of Japan (18590815 and 21590898 to YH) and a Grant in Aid of Japanese Arteriosclerosis Prevention Fund (to YH).

Competing interests None declared.

Patient and public involvement Patients and/or the public were not involved in the design, or conduct, or reporting, or dissemination plans of this research.

Patient consent for publication Not required.

Ethics approval The study protocol was registered in the University Hospital Medical Information Network Clinical Trials Registry (UMIN000012950). The protocol of this study conforms to the ethical guidelines of the 1975 Declaration of Helsinki and was approved by the ethical committee of Hiroshima University.

Provenance and peer review Not commissioned; externally peer reviewed.

Data availability statement Data are available on reasonable request. All data generated analysed during the current study are avilable from the corresponding author on reasonable request.

Open access This is an open access article distributed in accordance with the Creative Commons Attribution Non Commercial (CC BY-NC 4.0) license, which permits others to distribute, remix, adapt, build upon this work non-commercially, and license their derivative works on different terms, provided the original work is properly cited, appropriate credit is given, any changes made indicated, and the use is non-commercial. See: http://creativecommons.org/licenses/by-nc/4.0/.

ORCID iD

Yukihito Higashi http://orcid.org/0000-0001-5813-3672

\section{REFERENCES}

1 Gordon T, Castelli WP, Hjortland MC, et al. High density lipoprotein as a protective factor against coronary heart disease. The Framingham study. Am J Med 1977;62:707-14.

2 Gordon DJ, Probstfield JL, Garrison RJ, et al. High-Density lipoprotein cholesterol and cardiovascular disease. four prospective American studies. Circulation 1989;79:8-15.

3 Kitamura A, Iso H, Naito Y, et al. High-Density lipoprotein cholesterol and premature coronary heart disease in urban Japanese men. Circulation 1994:89:2533-9.

4 Saito I, Yamagishi K, Kokubo Y, et al. Association of high-density lipoprotein cholesterol concentration with different types of stroke and coronary heart disease: the Japan public health Center-Based prospective (JPHC) study. Atherosclerosis 2017;265:147-54.

5 Zhang Y, Tuomilehto J, Jousilahti P, et al. Total and high-density lipoprotein cholesterol and stroke risk. Stroke 2012;43:1768-74.

6 Yuhanna IS, Zhu Y, Cox BE, et al. High-Density lipoprotein binding to scavenger receptor-BI activates endothelial nitric oxide synthase. Nat Med 2001;7:853-7.
7 Nofer J-R, van der Giet M, Tölle M, et al. Hdl induces NO-dependent vasorelaxation via the lysophospholipid receptor S1P3. J Clin Invest 2004;113:569-81.

8 Cockerill GW, Huehns TY, Weerasinghe A, et al. Elevation of plasma high-density lipoprotein concentration reduces interleukin-1-induced expression of E-selectin in an in vivo model of acute inflammation. Circulation 2001;103:108-12.

9 Nofer J-R, Geigenmüller S, Göpfert C, et al. High density lipoproteinassociated lysosphingolipids reduce E-selectin expression in human endothelial cells. Biochem Biophys Res Commun 2003;310:98-103.

10 Riwanto M, Landmesser U. High density lipoproteins and endothelial functions: mechanistic insights and alterations in cardiovascular disease. J Lipid Res 2013;54:3227-43.

11 Zhu W, Saddar S, Seetharam D, et al. The scavenger receptor class B type I adaptor protein PDZK1 maintains endothelial monolayer integrity. Circ Res 2008;102:480-7.

12 de la Llera Moya M, McGillicuddy FC, Hinkle CC, et al. Inflammation modulates human $\mathrm{HDL}$ composition and function in vivo. Atherosclerosis 2012;222:390-4.

13 McGillicuddy FC, de la Llera Moya M, Hinkle CC, et al. Inflammation impairs reverse cholesterol transport in vivo. Circulation 2009;119:1135-45.

14 Ortiz-Munoz G, Couret D, Lapergue B, et al. Dysfunctional HDL in acute stroke. Atherosclerosis 2016;253:75-80.

15 Hirata A, Sugiyama D, Watanabe M, et al. Association of extremely high levels of high-density lipoprotein cholesterol with cardiovascular mortality in a pooled analysis of 9 cohort studies including 43,407 individuals: the EPOCH-JAPAN study. J Clin Lipidol 2018;12:674-84.

16 El Khoudary SR, Ceponiene I, Samargandy S, et al. Hdl (high-density lipoprotein) metrics and atherosclerotic risk in women. Arterioscler Thromb Vasc Biol 2018;38:2236-44.

17 El Khoudary SR, Wang L, Brooks MM, et al. Increase HDL-C level over the menopausal transition is associated with greater atherosclerotic progression. J Clin Lipidol 2016;10:962-9.

18 Keidar S, Bogner I, Gamliel-Lazarovich A, et al. High plasma highdensity lipoprotein levels, very low cardiovascular risk profile, and subclinical carotid atherosclerosis in postmenopausal women. $J$ Clin Lipidol 2009;3:345-50.

19 Barter PJ, Caulfield M, Eriksson M, et al. Effects of torcetrapib in patients at high risk for coronary events. N Engl J Med 2007;357:2109-22.

20 Schwartz GG, Olsson AG, Abt M, et al. Effects of dalcetrapib in patients with a recent acute coronary syndrome. $N$ Engl $J$ Med 2012;367:2089-99.

21 Lincoff AM, Nicholls SJ, Riesmeyer JS, et al. Evacetrapib and cardiovascular outcomes in high-risk vascular disease. N Engl J Med 2017;376:1933-42

22 Rohatgi A, Khera A, Berry JD, et al. Hdl cholesterol efflux capacity and incident cardiovascular events. N Engl J Med 2014;371:2383-93.

23 Khera AV, Demler OV, Adelman SJ, et al. Cholesterol efflux capacity, high-density lipoprotein particle number, and incident cardiovascular events: an analysis from the Jupiter trial (justification for the use of statins in prevention: an intervention trial evaluating rosuvastatin). Circulation 2017;135:2494-504

24 Ross R. Atherosclerosis--an inflammatory disease. N Engl J Med 1999;340:115-26.

25 Higashi Y, Noma K, Yoshizumi M, et al. Endothelial function and oxidative stress in cardiovascular diseases. Circ J 2009;73:411-8.

26 Inaba Y, Chen JA, Bergmann SR. Prediction of future cardiovascular outcomes by flow-mediated vasodilatation of brachial artery: a metaanalysis. Int J Cardiovasc Imaging 2010;26:631-40.

27 Matsuzawa Y, Kwon T-G, Lennon RJ, et al. Prognostic value of flow-mediated vasodilation in brachial artery and fingertip artery for cardiovascular events: a systematic review and meta-analysis. J Am Heart Assoc 2015;4:JAHA.115.002270.

$28 \mathrm{Li} \mathrm{XP}$, Zhao SP, Zhang XY, et al. Protective effect of high density lipoprotein on endothelium-dependent vasodilatation. Int J Cardiol 2000;73:231-6.

29 Spieker LE, Sudano I, Hürlimann D, et al. High-Density lipoprotein restores endothelial function in hypercholesterolemic men. Circulation 2002;105:1399-402.

30 Takaeko Y, Matsui S, Kajikawa M, et al. Association of extremely high levels of high-density lipoprotein cholesterol with endothelial dysfunction in men. J Clin Lipidol 2019;13:e661:664-72.

31 Tomiyama $\mathrm{H}$, Kohro $\mathrm{T}$, Higashi $\mathrm{Y}$, et al. A multicenter study design to assess the clinical usefulness of semi-automatic measurement of flow-mediated vasodilatation of the brachial artery. Int Heart $J$ 2012;53:170-5

32 American Diabetes Association. Clinical practice recommendations 1999. Diabetes Care 1999;22:S1-114. 
33 Expert Panel on Detection, Evaluation, and Treatment of High Blood Cholesterol in Adults. Executive summary of the third report of the National cholesterol education program (NCEP) expert panel on detection, evaluation, and treatment of high blood cholesterol in adults (adult treatment panel III). JAMA 2001;285:2486-97.

34 Wilson PW, D'Agostino RB, Levy D, et al. Prediction of coronary heart disease using risk factor categories. Circulation 1998;97:1837-47.

35 Hirata A, Okamura T, Sugiyama D, et al. The relationship between very high levels of serum high-density lipoprotein cholesterol and cause-specific mortality in a 20-year follow-up study of Japanese general population. J Atheroscler Thromb 2016;23:800-9.

36 Moriyama Y, Okamura T, Inazu A, et al. A low prevalence of coronary heart disease among subjects with increased high-density lipoprotein cholesterol levels, including those with plasma cholesteryl ester transfer protein deficiency. Prev Med 1998;27:659-67.

37 Takaeko Y, Matsui S, Kajikawa M, et al. Association of extremely high levels of high-density lipoprotein cholesterol with endothelial dysfunction in men. J Clin Lipidol 2019;13:664-72.

38 Alberti KG, Zimmet PZ. Definition, diagnosis and classification of diabetes mellitus and its complications. Part 1: diagnosis and classification of diabetes mellitus provisional report of a who consultation. Diabet Med 1998;15:539-53.

39 Matsuzawa Y. The definition and criteria of metabolic syndrome. Nihon Naika Gakkai Zasshi 2005;94:794-809.

40 Castelli WP, Garrison RJ, Wilson PW, et al. Incidence of coronary heart disease and lipoprotein cholesterol levels. The Framingham study. JAMA 1986;256:2835-8.

41 Inazu A, Brown ML, Hesler CB, et al. Increased high-density lipoprotein levels caused by a common cholesteryl-ester transfer protein gene mutation. N Engl J Med 1990;323:1234-8.

42 Maruyama T, Sakai N, Ishigami M, et al. Prevalence and phenotypic spectrum of cholesteryl ester transfer protein gene mutations in Japanese hyperalphalipoproteinemia. Atherosclerosis 2003;166:177-85.

43 Papp AC, Pinsonneault JK, Wang D, et al. Cholesteryl ester transfer protein (CETP) polymorphisms affect mRNA splicing, HDL levels, and sex-dependent cardiovascular risk. PLoS One 2012;7:e31930.

44 Ikewaki K, Mabuchi $\mathrm{H}$, Teramoto $\mathrm{T}$, et al. Association of cholesteryl ester transfer protein activity and TaqIB polymorphism with lipoprotein variations in Japanese subjects. Metabolism 2003;52:1564-70.

45 Gong M, Wilson M, Kelly T, et al. Hdl-Associated estradiol stimulates endothelial NO synthase and vasodilation in an SR-Bl-dependent manner. J Clin Invest 2003;111:1579-87.

46 Rosenson RS, Brewer HB, Ansell BJ, et al. Dysfunctional HDL and atherosclerotic cardiovascular disease. Nat Rev Cardiol 2016;13:48-60.

47 Srivastava RAK. Dysfunctional HDL in diabetes mellitus and its role in the pathogenesis of cardiovascular disease. Mol Cell Biochem 2018;440:167-87.

48 Kratzer A, Giral H, Landmesser U. High-Density lipoproteins as modulators of endothelial cell functions: alterations in patients with coronary artery disease. Cardiovasc Res 2014;103:350-61.

49 Vaisar T, Couzens E, Hwang A, et al. Type 2 diabetes is associated with loss of HDL endothelium protective functions. PLoS One 2018;13:e0192616.

50 Shaw LJ, Bugiardini R, Merz CNB. Women and ischemic heart disease: evolving knowledge. J Am Coll Cardiol 2009;54:1561-75.

51 Ernst N, Fisher M, Smith W, et al. The association of plasma high-density lipoprotein cholesterol with dietary intake and alcohol consumption. the lipid research clinics prevalence study. Circulation 1980;62:Iv41-52. 